\title{
An experimental investigation of the effects of gas solubility on the properties of horizontal slug flow
}

\author{
G.R. Magalhães ${ }^{a}$, G.F.N. Gonçalves ${ }^{b}$, J.B.R. Loureiro ${ }^{c, b, *}$, A.P. Silva Freire ${ }^{c}$ \\ ${ }^{a}$ Research Center, Brazilian Petroleum Company (Petrobras), Brazil \\ ${ }^{\mathrm{b}}$ Mechanical Engineering Department (DEM/Poli/UFRJ), C.P. 68526, 21941-972 Rio de Janeiro, Brazil \\ ${ }^{\mathrm{c}}$ Mechanical Engineering Program (PEM/COPPE/UFRJ), C.P. 68503, 21941-972 Rio de Janeiro, Brazil
}

\section{A R T I C L E I N F O}

Article history:

Received 27 March 2012

Received in revised form 8 August 2012

Accepted 1 October 2012

Available online 30 October 2012

\section{Keywords:}

Horizontal slug flow

Solubility

PIV

\begin{abstract}
A B S T R A C T
The present work investigates the effects of gas solubility on the fluid dynamic properties of a horizontal two-phase slug flow. Measurements of the size distributions of the liquid slug and of the long bubbles are provided, as well as local measurements of the liquid velocity in the slug, the velocity in the liquid film, the velocity of propagation of the nose of the long bubbles and the pressure drop. These parameters were obtained through two optical techniques: Shadow Sizer (SS) and Particle Image Velocimetry (PIV). The experiments indicate that the solubility has a substantial influence on the flow properties resulting, in particular, in a significant reduction of the local pressure gradient over a relatively short distance $(8 \mathrm{~m})$. The data are compared with modified versions of the mechanistic models of Dukler and Hubbard (Dukler, A.E., Hubbard, M., 1975, A model for gas-liquid slug flow in horizontal and near horizontal tubes, Ind. Eng. Chem. Fund. 14(4), 337-347) and of Orell (Orell, A., 2005, Experimental validation of a simple model for gas-liquid slug flow in horizontal pipes, Chem. Eng. Sci. 60, 1371-1381) so that the solubility effects can be taken into account.
\end{abstract}

(c) 2012 Elsevier Ltd. All rights reserved.

\section{Introduction}

In Brazil, about $90 \%$ of the oil and gas fields are located in deep waters (Silva et al., 2004). The resulting harsh conditions in which drilling and production operations take place imply that almost every conceivable type of multiphase flow can be found throughout the exploitation and production systems. Fluids that are multicomponent mixtures with complex phase behavior may flow in a disordered, highly intermittent manner, in patterns where the spatial distribution of the phases is unknown and difficult to specify quantitatively.

The demands for the economical and safe exploration of wells have favored the development of many predictive models for gas-liquid flows. These models normally rely on empirical or mechanistic arguments or on a combination of both (see e.g. Taitel and Barnea (1990a), Dukler and Fabre (1994), and Orell (2005), for a critical review). Of course, full numerical solutions of the differential equations of motion could also be attempted. However, the theoretical complications and numerical costs are prohibitive.

One particular new concern associated with oil production in ultradeep off-shore wells regards the effects that the solubility of a gas in a liquid may have on the properties of two-phase slug

\footnotetext{
* Corresponding author at: Mechanical Engineering Department (DEM/Poli/ UFRJ), C.P. 68526, 21941-972, Rio de Janeiro, Brazil.

E-mail address: jbrloureiro@gmail.com (J.B.R. Loureiro).
}

flows. In fact, in the Brazilian pre-salt scenario, a large influx of a soluble gas into the wellbore is possible to occur either because of the natural high concentrations of $\mathrm{CO}_{2}$ in discovered reservoirs or as a result of the use of processes for enhanced oil recovery. Gas injection into reservoirs has also been foreseen for sequestration of $\mathrm{CO}_{2}$ (Gerritsen and Durlofsky, 2005). Owing to fluid injection, profound changes in the well flow pattern and, consequently, in all of its properties can be induced.

The purpose of the present work is to investigate experimentally the changes in slug flow properties provoked by gas solubility and show how a very simple correction can be implemented to the existing classical mechanistic models so as to provide reliable theoretical predictions. In addition to the obvious need of designers and operators to correctly estimate pressure losses and fundamental frequencies associated to multiphase systems, the residence time of slugs in separators and other equipment must be properly determined. For example, subsea pumps operating on void fraction rates higher than the specified level can be permanently damaged.

The present working fluids are water and carbon dioxide (Carroll et al., 1991). Local measurements are provided by two optical techniques: Shadow Sizer (SS) and Particle Image Velocimetry (PIV). Shown data include the size distributions of the liquid slug and of the long bubbles, the liquid velocity in the slug, the velocity in the liquid film, the velocity of propagation of the nose of the long bubbles and the pressure drop. Experiments are used to generate two correlations that are subsequently incorporated into the 
mechanistic horizontal slug flow models of Dukler and Hubbard (1975) and Orell (2005).

The essential properties of gas-liquid flows are related to their configurations, frequently characterized through flow pattern maps. This method of analysis permits the fundamental physics of a particular configuration to be modeled in terms of idealized representations, which are expected to occur in problems where the same flow dynamics is retained. In the present work, two flow conditions were particularly selected to represent bubbly and slug flows. These conditions were defined with the help of the Shadow Sizer system. The statistics of the bubbles were obtained thorough the individual processing of 5500 images.

One of the central tenets of science is verifiability. The two slug flow models just mentioned are physically sound, very robust, vastly known and easy to implement. For these motives, they have been chosen as a natural support to the developments herein described. Predictions yielded by the modified models are compared with experimental measurements. The effects of solubility are shown to exert a significant influence on the flow properties.

\section{Experimental set up and measurement techniques}

\subsection{Experimental set up}

The experiments were conducted in an 8-m long acrylic pipe with 19-mm internal diameter. A schematic diagram of the working system is shown in Fig. 1. The water was driven by a centrifugal pump to a mixer where the gas phase (either air or carbon dioxide) was received from a pressure vessel. A system of valves was used to regulate and stabilize the flow rates. Absolute pressure was measured with a DPharp manometer, model EJA530A/HAC, from Yokogawa Electric Corporation. Typical measurement uncertainty is $0.075 \%$ of span.

Calibrated rotameters were used to measure the flow rates of both phases with typical uncertainties of $2 \%$ of span. Two acrylic boxes filled with water were installed at the test sections to minimize optical distortion. The hydraulic circuit was open; after leaving the second test section both phases were disposed to a drain.

\subsection{Measurement techniques}

\subsubsection{PIV measurements}

The PIV measurements were performed with a two-dimensional Dantec system. The light source was furnished by a double pulsed Nd:YAG laser that produced short duration (4 ns) high energy (135 mJ) pulses of green light $(532 \mathrm{~nm})$. The collimated laser beam was transmitted through a series of cylindrical and spherical lenses to generate a $1 \mathrm{~mm}$ thick lightsheet. The reflected light was recorded at $15 \mathrm{~Hz}$ by a CCD camera with $1280 \times 1024$ pixels and 12-bit resolution. The camera was fitted with a Nikkor $60 \mathrm{~mm} f / 2.8 \mathrm{D}$ lens. Image calibration was made by taking pictures of a reference target specially designed for the present purpose.

For all measurements, computational conditions for the velocity vectors were fixed. Adaptive correlation (Dynamic Studio Software v.3.14) has been processed on $32 \times 32$ pixels-size final interrogation windows, with $50 \%$ overlap. The pixel resolution is $6.45 \times 6.45 \mu \mathrm{m}$. Particle image treatment used subpixel cell shifting and deformation, to allow bias and random error reduction. A widely accepted estimation of the absolute displacement error using these algorithms is 0.05 pixels. Different thresholds including signal-to-noise ratio and velocity vector magnitude were used as post-processing steps.

The PIV seeding was made with fluorescent particles that reflect light with a $590 \mathrm{~nm}$ wavelength. Using an appropriate red filter, laser reflections caused by the surface of the bubbles could be totally blocked so as to permit only the passage of the light emitted by the $10 \mu \mathrm{m}$ diameter Rhodamine particles.

The measurement accuracy of PIV results is widely known to depend on a variety of aspects extending from set up recording conditions to the numerical methods used for calculations. The choice of particle image diameter, particle image shift, image density, image quantization levels, background noise and velocity gradients are only a few of the aspects that deserve careful attention during the conduction of a PIV experimental campaign.

According to Adrian (1997), regardless of the source, there is a root mean square error in determining the displacement of the centroid of the particle image, $\sigma_{\Delta x}$. Considering that the uncertainty of the time between pulses $\Delta t$ is irrelevant for low velocity flows, the rms uncertainty in the velocity measurement can be given by:

$\sigma_{u}=\frac{\sigma_{\Delta x}}{\Delta t}=\frac{\sigma_{\Delta X}}{M_{0} \Delta t}$

where the subscript $x$ denotes the position of the seeding particle in the fluid, $X$ is the corresponding position in the image plane and $M_{0}$ is the image magnification. The rms uncertainty of the displacement in the pixel plane can be written as $\sigma_{\Delta X}=c_{\tau} d_{\tau}$, with $d_{\tau}^{2}=d_{e}^{2}+d_{r}^{2}$, where $d_{\tau}$ is the diameter of the recorded image particle and $c_{\tau}$ is a constant that depends on the ability of the analysis procedure to determine the displacement between consecutive images. The recorded image diameter $d_{\tau}$ is then a function of the resolution of the recording medium $\left(d_{r}\right)$ and of the diameter of the optical image prior to being recorded $\left(d_{e}\right)$. Considering a Gaussian intensity distribution of the geometric image of the particle and diffracted limited imaging, the diameter of the diffracted image of the particle can be written as $d_{e}^{2}=M_{0} d_{p}^{2}+\left(2.44\left(1+M_{0}\right) f^{\#} \lambda\right)^{2}$.

For the present experimental conditions, $f^{\#}=4.0$ is the $f$-number of the imaging lens, $\lambda=532 \mathrm{~nm}$ is the wavelength of the laser and the particle diameter, $d_{p}$, is $10 \mu \mathrm{m}$. $d_{r}$ is taken to be equal to the pixel resolution, $6.45 \mu \mathrm{m}, M_{0}=0.056, \Delta t=150 \mu \mathrm{s}$. Considering

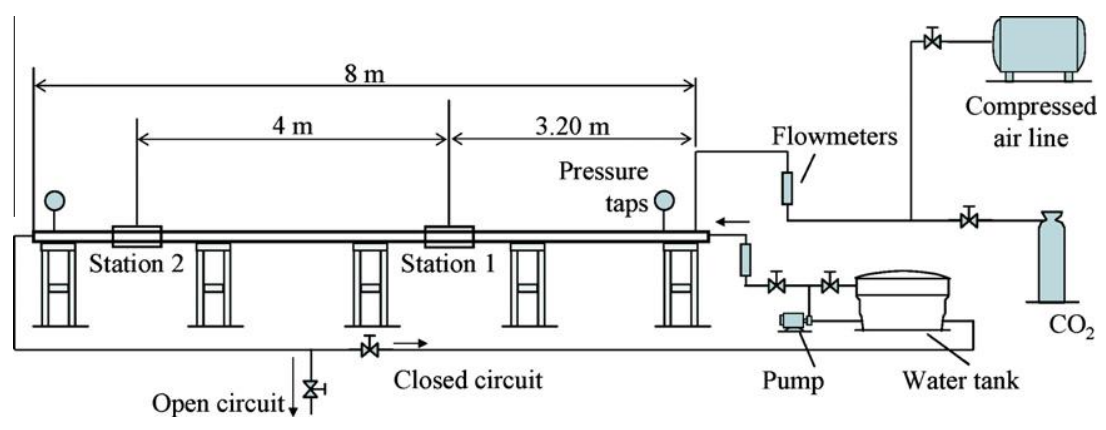

Fig. 1. Schematic diagram of the experimental set up. 
$c_{\tau}=0.04$, as suggested by Adrian (1997), the above equations furnish a systematic velocity uncertainty of $\sigma_{\Delta X}=0.04 \mathrm{~ms}^{-1}$.

The total number of samples for every point of velocity measurement was adjusted to ensure statistical independence between consecutive measurements as well as to furnish an interval of confidence of 95\%. The random uncertainty $\epsilon$ was calculated according to ANSI/ASME Standards using a $t$-distribution and the standard deviation of the measured values $\left(\epsilon=t u_{r m s} / \sqrt{N}\right.$, where $N$ equals the total number of samples). As described in Coleman and Steele (2009), the bias and random uncertainty can be combined into a single final number that represents the interval that contains the true values within $95 \%$ confidence. The highest observed combined uncertainty was $0.09 \mathrm{~ms}^{-1}$. The combined uncertainty is also shown as error bars in the velocity distributions presented below.

\subsubsection{SS measurements}

To further inspect the two-phase flow characteristics, a Shadow Sizer (Dantec Dynamics) measurement system was used. The system consists of a high speed camera (NanoSense MK III) and a constellation led back light that provided high resolution images $(1280 \times 1024$ pixels $)$ at $1000 \mathrm{~Hz}$ frame rate. The software Dynamic Studio was responsible for triggering the camera and the illumination system, as well as detecting contour differences on the images to provide statistical results about the flow. Additional image handling and processing was made with programs specially implemented in the software Mathematica ${ }^{\mathrm{TM}}$.

The Shadow Sizer images were subjected to different brightness and contrast treatments to make the contour of the bubbles sharply defined. Fig. 2 shows two typically post-processed images with the bubble contour highlighted in yellow and the circumscribed rectangle in red.

Given the highly intermittent pattern of two phase slug flows, the main source of uncertainty for bubble size and slug length measurements stems from the actual random variation observed in the flow field. The overall bias uncertainty related to the image resolution, typically of the order of few microns, can then be considered irrelevant. In analogy to the procedure described above for the PIV measurements, the maximum uncertainty observed in length calculations was $9.74 \mathrm{~mm}$ for the $\mathrm{CO}_{2}$-water flow at Station 2 .

\subsubsection{Image processing}

The application of PIV to multiphase systems might incur in problems that are absent in single phase flows. Since information related to the different phases must be separated, special techniques may have to be devised.

In the present work, velocity profiles of the continuous phase were obtained from interrogation windows that were completely free of bubbles. In the liquid slug, the low concentration of bubbles (Fig. 2) resulted in the rejection of less than $5 \%$ of images. Fluores- cent particles together with a combination of optical filters can be used to determine the velocity field of the continuous phase close to walls or to gas-liquid interfaces (Sridhar and Katz, 1995). In the liquid film, the area occupied by the liquid needed not to be determined through any special technique. A standard post-processing procedure was used.

\section{Results}

Slug flows are very complex flows characterized by strong pressure, velocity and phase fraction fluctuations (Netto et al., 1999; Nydal et al., 1992). A useful and relatively simple way to model these flows is to consider the concept of a unit cell, whereby the flow is seen as a succession of bubbles and liquid slugs that are periodic in time and space. Several works can be identified in literature where this line of thought is pursued (see, e.g., the reviews by Taitel and Barnea (1990a, 1994); or the more recent work of Orell (2005)). The work of Dukler and Hubbard (1975) is generally considered the first to propose a comprehensive model for the dynamic bahaviour of horizontal gas-liquid slug flow. The flow structure in described by sixteen variables, four physical properties of the flow and two geometric parameters that characterize pipe size and orientation. Since only 12 independent equations are furnished to determine a solution, four variables or additional closure relations must be given to permit calculations.

Nicholson et al. (1978) proposed some modifications and extensions to the model of Dukler and Hubbard (1975), in terms of new equations for the average translational velocity of the unit cell and the liquid fraction in the slug $\left(R_{S}\right)$, an input quantity. To render these models a complete predictive tool, a second input quantity, the slug flow frequency $\left(v_{t}\right)$, needs to be specified. Considering empirical and mechanistic arguments, many expressions for $v_{t}$ have been proposed by various authors. For instance, Zabaras (2000) describes in detail eight of those expressions (including his own), comparing their predictions with data collected in a laboratory flow loop and available in the literature; 339 points were considered covering pipe diameters from 1 to 8 inches. In a more recent work, Al-Safran (2009) uses an extended experimental database to propose a new empirical correlation; eight other correlations are discussed in his work.

The work of Orell (2005) reformulates the simplified model of Taitel and Barnea (1990b), including an additional pressure loss term due to Cook and Behnia (2000). Taitel and Barnea (1990b) approximated the variable liquid film thickness by a uniform layer and used a momentum balance over the slug unit to estimate the pressure losses, which for horizontal slugs were shown to have a frictional character.

Here, results are presented in terms of the relevant variables originally introduced by Dukler and Hubbard (1975). In fact, to
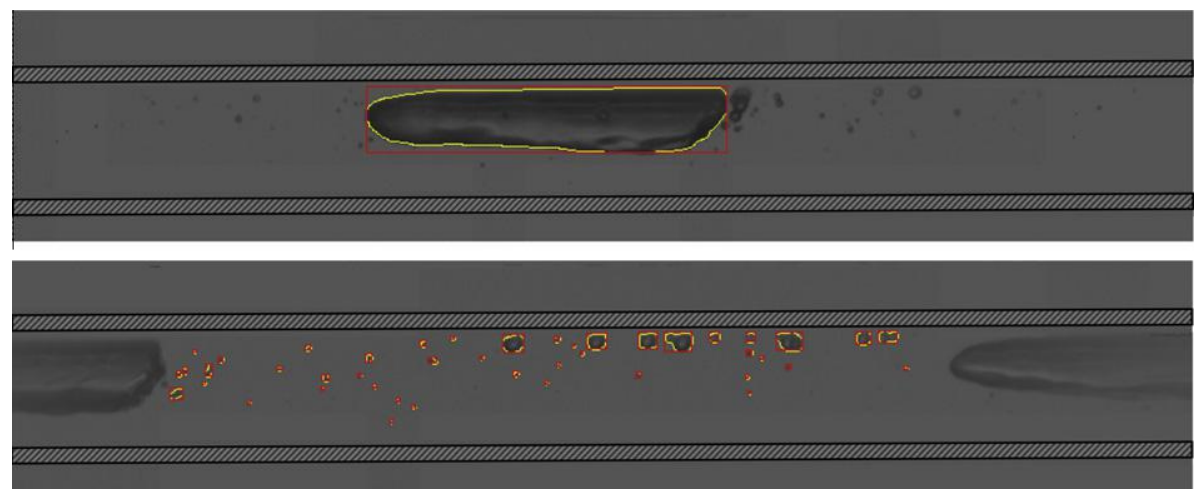

Fig. 2. Typical Shadow Sizer images after post-processing. 
allow a comparative analysis of the effects of the solubility of carbon dioxide in water on the flow properties, experiments were also carried out with an air-water mixture.

\subsection{Shadow Sizer (SS) results for bubbly flow}

Before the effects of gas solubility on slug flow were accounted for, a sensitivity analysis of the proposed conditions for the experiments was made. Measurements in the bubbly flow regime showed an appreciable decrease in bubble size distribution between Stations 1 and 2 (Fig. 1), which were set 4-m apart. In the two run tests that were performed, images were recorded at $20 \mathrm{~Hz}$. At each station, 239 bubbles were received. Fig. 3 shows that between Stations 1 and 2, an appreciable decrease of $35 \%$ in average bubble volume occurred. The consolidated results are presented in Table 1.

\subsection{SS results for slug flow}

The slug flow was determined with a liquid phase (water) flow rate of $1000 \mathrm{lh}^{-1}$ and a gas flow rate (air or carbon dioxide) of $315 \mathrm{lh}^{-1}$. Typically about 130 unit cells were observed during a certain experimental run. The SS statistics were obtained from images that were recorded at a rate of $100 \mathrm{~Hz}$.

The notation chosen here is that of Dukler and Hubbard (1975). The following parameters were estimated with the SS system: slug frequency $\left(v_{t}\right)$, slug length $\left(L_{s}\right)$, length of liquid film $\left(L_{f}\right)$, slug hold up $\left(R_{s}\right)$, film hold up just before pick up by a $\operatorname{slug}\left(R_{f e}\right)$, translational velocity of the unit cell $\left(V_{t}\right)$.

A typical result for the slug length of both flows of interest are shown in Fig. 4 for measurements taken at Station 1. The size and shape of slug bubbles are known to depend on many properties of the flow and on geometry and, in particular, their lengths are known to exhibit a large variation (standard deviation, $\sigma$ ) about a mean value $(\mu)$ (Fig. 5).

The probability density functions of film $\left(L_{f}\right)$ and slug $\left(L_{S}\right)$ lengths resulting from air-water and $\mathrm{CO}_{2}$-water flows at Stations 1 and 2 are shown in Fig. 5. For the air-water experiment, the dynamics of flow due to small changes in $v_{t}$ meant that the average values of $L_{f}$ increased about 5\% between stations (Fig. 5a), but $\sigma$ remained about the same. The effects of the solubility, however, provoke a shift to the left on the density function of $L_{f}$, implying that for the $\mathrm{CO}_{2}$-water flow, the average value of $L_{f}$ decreased about $4 \%$ between Stations 1 and 2 (Fig. $5 \mathrm{~b}$ ). The changes in $L_{s}$ shown in Fig. $5 c$ and $d$ are very pronounced, resulting solely from the changes in $v_{t}$. The changes on the bulk volume of the liquid slug due to solubility effects were insignificant because of the low concentration of small bubbles.
Table 1

Summary of Shadow Sizer results for bubble volume measurements in $\mathrm{CO}_{2}$-water bubbly flows: comparison between Stations 1 and 2 .

\begin{tabular}{lcc}
\hline & Station 1 & Station 2 \\
\hline Number of bubbles & 239 & 239 \\
Average volume $\left(\mathrm{mm}^{3}\right)$ & 130 & 85 \\
Total volume $\left(\mathrm{mm}^{3}\right)$ & 31,182 & 20,286 \\
\hline
\end{tabular}
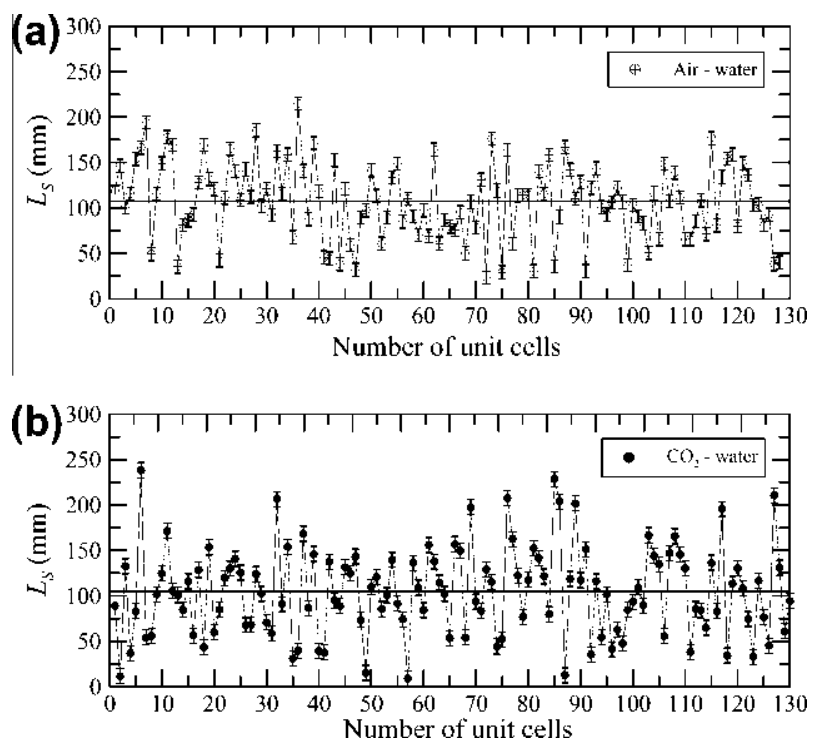

Fig. 4. Slug length $\left(L_{s}\right)$ results for: (a) air-water and (b) $\mathrm{CO}_{2}$-water flows at Station 1.

Observed changes in $v_{t}$ from Station 1 to Station 2 might be justified on the ground of transient effects. In our experiment, Station 1 was located at a distance of about $168 D$ from the entrance region. The initiation and evolution of slugs in a horizontal pipe were studied by Ujang et al. (2006). The downstream reduction in slug frequency was shown to be strongly dependent on gas and liquid velocities when experiments were carried out at atmospheric pressure. The development length for the various conditions ranged from $60 D$ to $180 D$.

All results obtained with the Shadow Sizer system are summarized in Table 2.

\subsection{PIV results for slug flow}

The PIV mean flow statistics were obtained from 1500 pairs of pictures, with a time interval between laser pulses of $150 \mu$ s. The

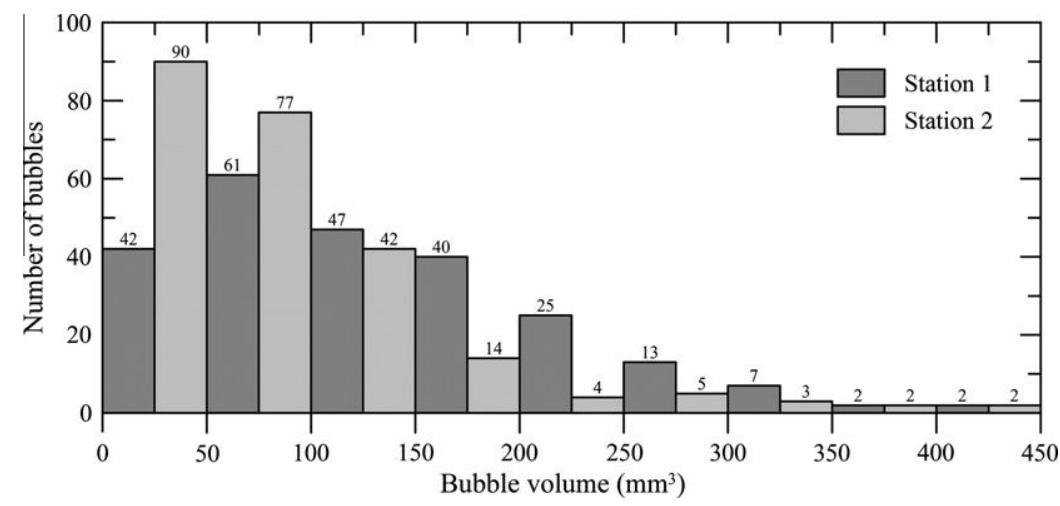

Fig. 3. Bubble size distribution for a $\mathrm{CO}_{2}$-water bubbly flow at Stations 1 and 2 . Water flow rate is $400 \mathrm{lh}^{-1}$. 

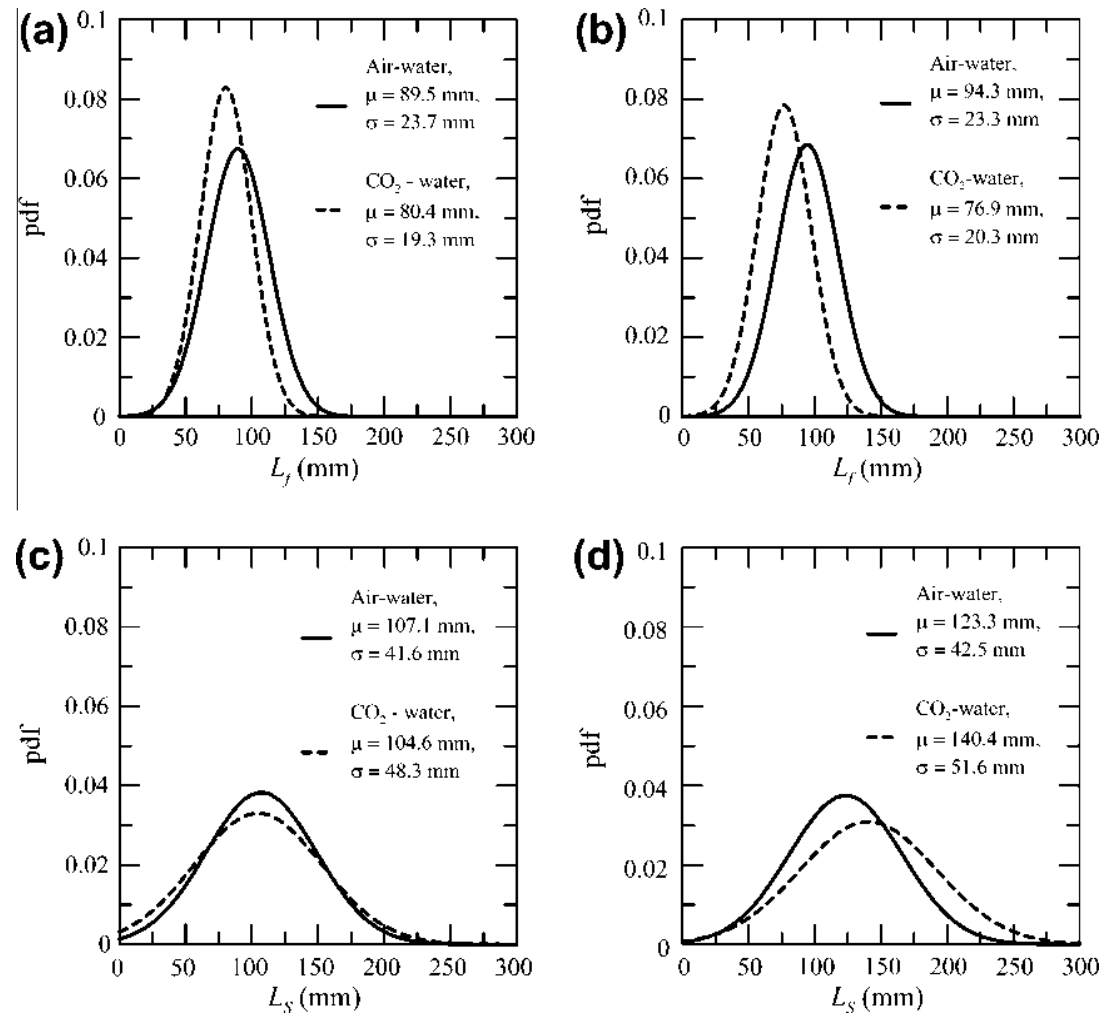

Fig. 5. Probability density functions of film and slug lengths ( $L_{f}$ and $L_{s}$ respectively) for air-water and $\mathrm{CO}_{2}$-water flows: (a and c) Station 1 , (b and d) Station 2 .

Table 2

Summary of Shadow Sizer results for air-water and $\mathrm{CO}_{2}$-water slug flows.

\begin{tabular}{lcccc}
\hline & Air, Station 1 & Air, Station 2 & $\mathrm{CO}_{2}$, Station 1 & $\mathrm{CO}_{2}$, Station 2 \\
\hline$v_{t}(\mathrm{~Hz})$ & 8.56 & 7.87 & 8.78 & 7.23 \\
$L_{s}(\mathrm{~mm})$ & 107.2 & 123.4 & 104.6 & 140.4 \\
$L_{f}(\mathrm{~mm})$ & 89.5 & 94.3 & 80.4 & 76.9 \\
$L_{u}(\mathrm{~mm})$ & 196.7 & 217.7 & 185.0 & 217.3 \\
$R_{s}$ & 0.98 & 0.99 & 0.99 & 0.99 \\
$R_{f e}$ & 0.26 & 0.25 & 0.32 & 0.34 \\
$V_{t}\left(\mathrm{~ms}^{-1}\right)$ & 1.68 & 1.71 & 1.62 & 1.56 \\
\hline
\end{tabular}

local velocity profiles of the mean velocity of fluid in the slug $\left(V_{s}\right)$ and of the mean velocity of fluid in the film in front of the slug $\left(V_{f e}\right)$ are shown in Figs. 6 and 7. The density functions of the mean velocity profiles averaged over a cross section are also shown.

The average value of $V_{S}$ for the air-water and $\mathrm{CO}_{2}$-water flows at Station 1 were 1.42 and $1.39 \mathrm{~ms}^{-1}$ respectively. At the same station, $V_{f e}$ changed from 1.15 to $1.13 \mathrm{~ms}^{-1}$ respectively. These slight differences are due to the short distance between the point where $\mathrm{CO}_{2}$ was injected into the flow and the point were the measurements were made.

At Station 2, however, differences were more pronounced. The average value of $V_{S}$ for the air-water and $\mathrm{CO}_{2}$-water flows changed from 1.47 to $1.33 \mathrm{~ms}^{-1}$ whereas $V_{f e}$ changed from 1.14 to $1.11 \mathrm{~ms}^{-1}$. In the model of Dukler and Hubbard, $V_{s}$ is uniquely determined from the input mass flow rates, fluid densities and pipe diameter, being independent of the liquid distribution between the film and the slug. This assumption has been further corroborated by Nicholson et al. (1978).

The PIV results are summarized in Table 3.

\section{Rate of mass transfer at the interface between phases}

The amount of $\mathrm{CO}_{2}$ absorbed in the water slug was determined experimentally. To simplify the analysis, the saturation of water is not considered, total pressure is constant, the diffusional resistance is entirely in the liquid and the variation of the mass-transport coefficient, $k_{c}$, with bubble size is neglected.

Considering the surface area of the bubbles to be $\alpha_{2} V^{2 / 3}$, the mass transfer equation is written as

$-\frac{d V}{d t}=\frac{\alpha_{2} k_{c}}{H} V^{2 / 3}$

where $k_{c}$ ([LT $\left.\left.{ }^{-1}\right]\right), H$ is Henry's constant $(=1.06)$ and $\alpha_{2}(=3.7)$ was defined by the experimental data.

The determination of $k_{c}$ for bubbles even with a simple geometry may be exceedingly complicated. Correlations available in literature furnish values of $k_{c}$ in the range of $0.0015-0.010 \mathrm{~cm} \mathrm{~s}^{-1}$ for diameters between 0.2 and $5 \mathrm{~cm}$. Since the average value of $L_{f}$ shown in Table 2 is well over $5 \mathrm{~cm}$, we have here considered $k_{c}=0.0001 \mathrm{~ms}^{-1}$.

The following relation is also used

$l=\alpha_{1} V^{1 / 3}$.

The length and interfacial area of slug bubbles versus their volumes for the present flow conditions are shown in Fig. 8a and b. The resulting functional dependences were found to be $L_{f}=271$ $\left(V^{1 / 3}\right)^{2}-2.4 V^{1 / 3}$ and $A=3.7\left(V^{2 / 3}\right)$. To find the area of a large bubble, the simplest possible approach was used. The division of its projected area (Fig. 2) by total length gave the average height, $h$. With $h$, the average chord, $c$, of the liquid film was found together with the average cross sectional area of the bubble, $S$. The volume and total interfacial area of the bubble were then given by $\mathrm{Vol}=S L_{f}$ and Area $=2 S+c L_{f}$

\section{Theoretical predictions}

To implement the previous developments, the works of Dukler and Hubbard (1975) and Orell (2005) were chosen. As particular 

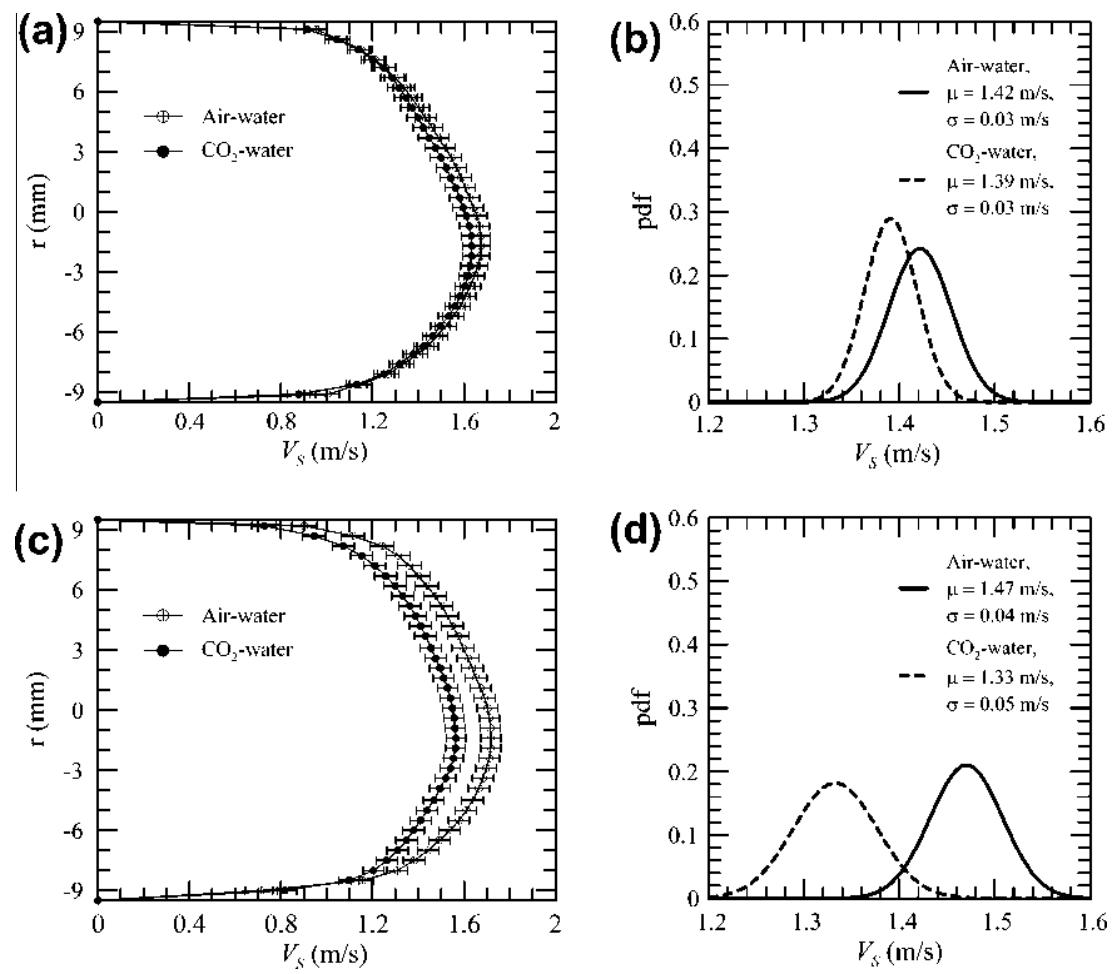

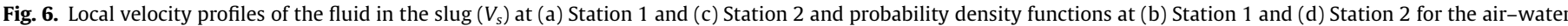
and $\mathrm{CO}_{2}$-water flows.
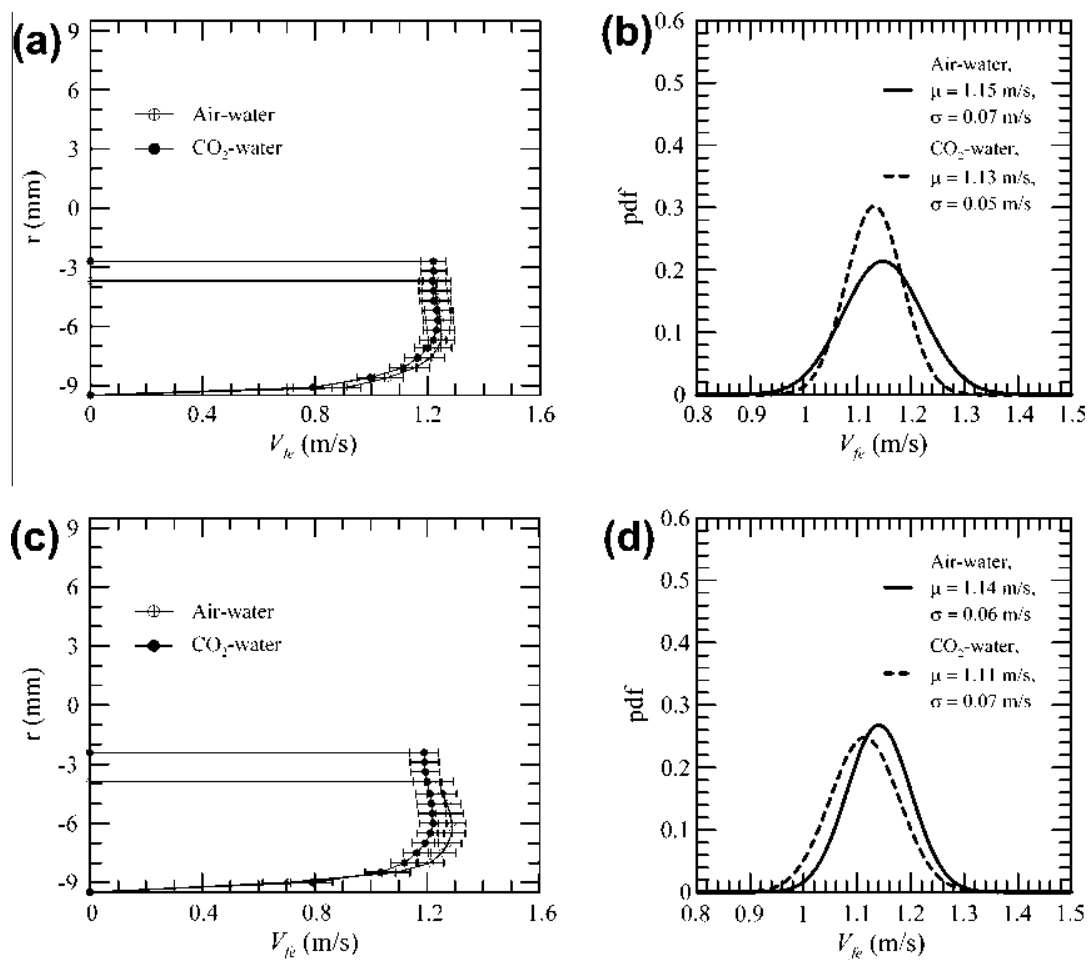

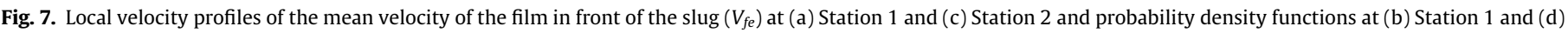
Station 2 for the air-water and $\mathrm{CO}_{2}$-water flows.

attributes, these models are very well known, are based on consistent physical hypotheses and can be easily implemented numerically. Empirical correlations for $R_{s}$ (liquid fraction in the slug) and $v_{t}$ (frequency of unit cell) that are required for use of these models are not a problem here. In fact, the present simulations were performed with data obtained directly from the experiments.

In a complementary note, we clarify that seven of the expressions quoted in Zabaras (2000) and Al-Safran (2009) were used 
Table 3

Summary of PIV results for air-water and $\mathrm{CO}_{2}$-water slug flows.

\begin{tabular}{lllll}
\hline & Air, Station 1 & Air, Station 2 & $\mathrm{CO}_{2}$, Station 1 & $\mathrm{CO}_{2}$, Station 2 \\
\hline$V_{s}\left(\mathrm{~ms}^{-1}\right)$ & 1.42 & 1.47 & 1.39 & 1.33 \\
$V_{f e}\left(\mathrm{~ms}^{-1}\right)$ & 1.15 & 1.14 & 1.13 & 1.11 \\
\hline
\end{tabular}

(a)
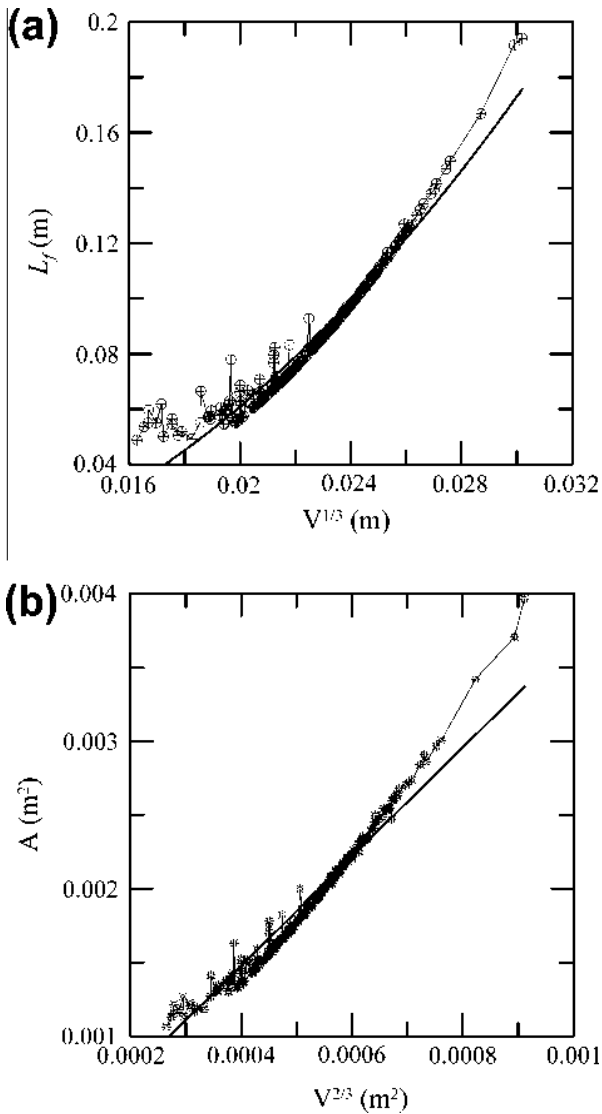

Fig. 8. Length and interfacial area of slug bubbles.

Table 4

Comparison between the present measurements and predictions given by the models of Dukler and Hubbard (1975) and Orell (2005), air-water flow. $v_{t}=7.87 ; R_{s}=0.991$.

\begin{tabular}{llll}
\hline & $\begin{array}{l}\text { Dukler and Hubbard } \\
(1975)\end{array}$ & $\begin{array}{l}\text { Orell } \\
(2005)\end{array}$ & $\begin{array}{l}\text { Exp. (Air, } \\
\text { Station 2) }\end{array}$ \\
\hline$v_{t}(\mathrm{~Hz})$ & - & - & 7.87 \\
$L_{s}(\mathrm{~mm})$ & 139.0 & 192.0 & 123.4 \\
$L_{f}(\mathrm{~mm})$ & 81.0 & 54.0 & 94.3 \\
$R_{f e}$ & 0.66 & - & 0.25 \\
$V_{s}\left(\mathrm{~ms}^{-1}\right)$ & 1.29 & 1.29 & 1.47 \\
$V_{f e}\left(\mathrm{~ms}^{-1}\right)$ & 0.98 & 0.10 & 1.14 \\
$V_{t}\left(\mathrm{~ms}^{-1}\right)$ & 1.59 & 1.78 & 1.71 \\
$\Delta p / \Delta x\left(\mathrm{~Pa} \mathrm{~m}^{-1}\right)$ & 1111 & 822 & 1200 \\
\hline
\end{tabular}

to estimate $v_{t}$. Predictions invariably gave results around 4 , an estimation much smaller than the observed values, of the order of 7 .

A comparison between the present air-water flow measurements and theoretical predictions furnished by the models of Dukler and Hubbard (1975) is shown in Table 4 for Station 2. Parameters $L_{u}$ and $V_{t}$ are predicted to within $1 \%$ and $7 \%$ accuracy and $\Delta P \mid \Delta x$ to within $8 \%$; however, a difference of about $14 \%$ is observed for the predictions of $L_{f}$. Results yielded by the model of Orell (2005) are also shown in Table 4. They predict $L_{u}$ and $V_{t}$ to within $13 \%$ and $4 \%$ respectively and $\Delta P / \Delta x$ to within $32 \%$. The model of Orell overestimates $L_{s}$ by $55 \%$.

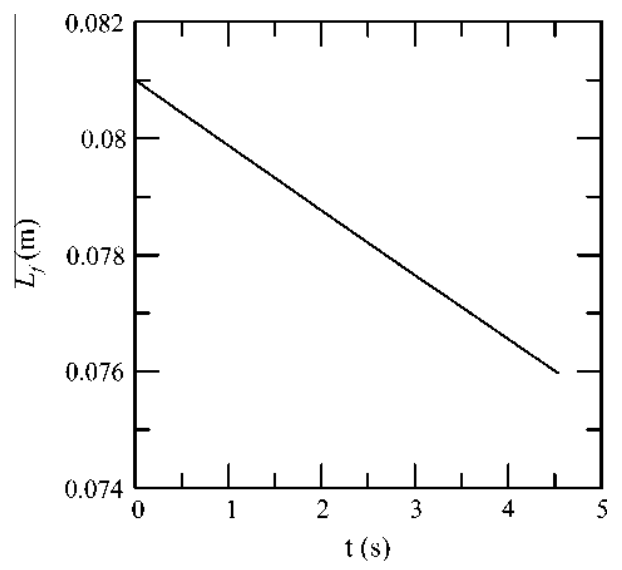

Fig. 9. Effects of gas solubility on $L_{f}$ according to Eq. (2).

Table 5

Comparison between the present measurements and predictions given by the modified models of Dukler and Hubbard (1975) and Orell (2005), $\mathrm{CO}_{2}$-water flow. $v_{t}=7.23 ; R_{s}=0.991$

\begin{tabular}{llll}
\hline & D\&H modified & Orell modified & Exp. $\left(\mathrm{CO}_{2}\right.$, Station 2$)$ \\
\hline$v_{t}(\mathrm{~Hz})$ & - & - & 7.23 \\
$L_{s}(\mathrm{~mm})$ & 139.2 & 190.3 & 140.4 \\
$L_{f}(\mathrm{~mm})$ & 76.0 & 51.8 & 76.9 \\
$R_{f e}$ & 0.47 & - & 0.34 \\
$V_{s}\left(\mathrm{~ms}^{-1}\right)$ & 1.29 & 1.29 & 1.33 \\
$V_{f e}\left(\mathrm{~ms}^{-1}\right)$ & 0.99 & 0.10 & 1.11 \\
$V_{t}\left(\mathrm{~ms}^{-1}\right)$ & 1.59 & 1.78 & 1.56 \\
$\Delta p / \Delta x\left(\mathrm{~Pa} \mathrm{~m}^{-1}\right)$ & 1070 & 819 & 1062 \\
\hline
\end{tabular}

Implementation of Eq. (2) into the models of Dukler and Hubbard (1975) and of Orell (2005) allows the effects of solubility to be accounted for in the properties of slug flow. The variation in length of the liquid film with time over the whole length of the test section is shown in Fig. 9.

A comparison between the present measurements and theoretical predictions furnished by the modified models of Dukler and Hubbard (1975) and Orell (2005) is shown in Table 5 for Station 2 . The variation in $v_{t}$ experimentally observed over Stations 1 and 2 and shown in Table 2 cannot be predicted by both models, which consider this parameter invariant in space and time. For the present simulations we have used $v_{t}=7.23$. Regarding the predictions of Dukler and Hubbard (1975), the predicted values of $L_{f}$, $L_{s}, V_{t}$ and $\Delta P / \Delta x$ all agree with the experimental data to within $5 \%$. The predictions given by the model of Orell (2005) underestimates $L_{f}$ by $33 \%$ and $\Delta P / \Delta x$ by $23 \%$.

The implementation of the solubility model requires the use of Eqs. (2) and (3), giving rise to a new algebraic equation that is time dependent and has to be implemented in the predictive models for the estimation of $L_{f}$. In fact, the models of Dukler and Hubbard and of Orell are initially run to furnish the initial flow conditions. Next, Eqs. (2) and (3) are used to provide the time evolution of the flow. The method is hence sensitive to choices of $k_{c}, \alpha_{1}$ and $\alpha_{2}$. The results of Table 5 show that the values presently assigned to these constants are consistent with the current experimental conditions.

\section{Conclusion}

This work has shown that the effects of gas solubility on the properties of slug flows are significant even for very short distances. We have shown that, for the present experimental conditions, the solubility of $\mathrm{CO}_{2}$ in water results in a large volume 
reduction of the gas phase. This, in turn, provokes a significant reduction in $\Delta P / \Delta x$.

A simple model for the rate of mass transfer at the interface between phases based on Eq. (2) is implemented in the mechanistic models of Dukler and Hubbard (1975) and Orell (2005). One of the models $(\mathrm{D} \& \mathrm{H})$ is shown to account very well for the predictions of $L_{f}$ and $\Delta P / \Delta x$, providing an agreement within $5 \%$.

In the experiments, a reduction of $4 \%$ in $L_{f}$ resulted in a reduction of $11.5 \%$ in $\Delta P / \Delta x$. In the simulations, a reduction of $6 \%$ in $L_{f}$ resulted in a reduction of $4 \%$ in $\Delta P / \Delta x$. A decrease in $L_{f}$ provokes a decrease in both the pressure drop due to friction across the slug and the pressure drop due to acceleration. In the numerical simulations, and over the considered length, these two effects resulted in a decrease of $\Delta P / \Delta x$.

The purpose of the present work has not been to propose a new model for the description of slug flows or even to review the existing models. Rather, we have striven to show that the effects of solubility are important and that the implementation of the simple model based on Eq. (2) provides good prediction for some flow properties. The present authors are currently working on new elaborations for the existing models so as to improve predictions. In any case, the present results give strong evidence that any model advanced to simulate two-phase flows with a soluble phase cannot safely ignore this feature.

\section{Acknowledgements}

Authors are grateful to Dr. A. Leibsohn of Petrobras, who suggested this investigation. In the course of the research, JBRL benefited from a CNPq Research Fellowship (Grant No. 301172/2010-2) and from further Financial Support through Grant 477354/2011-4. APSF is grateful to the Brazilian National Research Council (CNPq) for the award of a Research Fellowship (Grant No. 303982/2009-8). The work was financially supported by CNPq through Grants No.
477293/2011-5 and by the Rio de Janeiro Research Foundation (FAPERJ) through Grant E-26/102.937/2011.

\section{References}

Adrian, R.J., 1997. Dynamic ranges of velocity and spatial resolution of particle image velocimetry. Meas. Sci. Instrum. 8, 1393-1398.

Al-Safran, E., 2009. Investigation and prediction of slug frequency in gas/liquid horizontal pipe flow. J. Petrol. Sci. Eng. 69, 143-155.

Carroll, J., Slupsky, J., Mather, A., 1991. The solubility of carbon dioxide in water at low pressure. J. Phys. Chem. Ref. Data 20, 1201-1209.

Coleman, H.W., Steele, W.G., 2009. Experimentation, Validation and Uncertainty Analysis for Engineers. John Wiley \& Sons, 334p.

Cook, M., Behnia, M., 2000. Pressure drop calculation and modelling of inclined intermittent gas-liquid flow. Chem. Eng. Sci. 55, 4699-4708.

Dukler, A.E., Fabre, J., 1994. Gas liquid slug flow: knots and loose ends. In: Hewitt G.F., Kim, J.H., Lahey, R.T., Delhaye, J.M., Zuber, N. (Eds.), Multiphase Science and Technology. Two Phase Flow Fundamentals. Begell House, Wallinford.

Dukler, A.E., Hubbard, M., 1975. A model for gas-liquid slug flow in horizontal and near horizontal tubes. Ind. Eng. Chem. Fund. 14, 337-347.

Gerritsen, M.G., Durlofsky, L.J., 2005. Modeling fluid flow in oil reservoirs. Annu. Rev. Fluid Mech. 37, 211-238.

Netto, J., Fabre, J., Peresson, L., 1999. Shape of long bubbles in horizontal slug flow. Int. J. Multiphase Flow 25, 1129-1160.

Nicholson, M., Aziz, K., Gregory, G., 1978. Intermittent two phase flow in horizontal pipes: predictive models. Can. J. Chem. Eng. 56, 653-663.

Nydal, O., Pintus, S., Aadreussi, P., 1992. Statistical characterization of slug flow in horizontal pipes. Int. J. Multiphase Flow 18, 439-453.

Orell, A., 2005. Experimental validation of a simple model for gas-liquid slug flow in horizontal pipes. Chem. Eng. Sci. 60, 1371-1381.

Silva, C.T., Mariolani, J.R.L., Boonet, E.J., Lomba, R.F.T., Santos, O.L.A., Ribeiro, P.R., 2004, Gas solubility in synthetic fluids: a well control issue. In: SPE Annual Technical Conference and Exhibition. Houston, SPE 91009.

Sridhar, G., Katz, J., 1995. Drag and lift forces on microscopic bubbles entrained by a vortex. Phys. Fluids 7, 389-399.

Taitel, Y., Barnea, D., 1990a. Two-phase slug flow. Adv. Heat Transfer 20, 83-132.

Taitel, Y., Barnea, D., 1990b. A consistent approach for calculating pressure drop in inclined slug flow. Chem. Eng. Sci. 45, 1191-1206.

Ujang, P.M., Lawrence, C.J., Hale, C.P., Hewitt, G.F., 2006. Slug initiation and evolution in two-phase horizontal flow. Int. J. Multiphase Flow 32, 527-552.

Zabaras, G.J., 2000. Predictions of slug frequency for gas/liquid flows. SPE J. 5, 252 258. 